INPLASY

PROTOCOL

To cite: Luo et al. Application of endometrial receptivity array (ERA) in embryo transfer cycles: a systematic review and meta-analysis. Inplasy protocol 202190013. doi: 10.37766/inplasy2021.9.0013

Received: 05 September 2021

Published: 05 September 2021

Corresponding author:

Yuanjiao Liang

yuanjiao1965@126.com

Author Affiliation:

Zhongda Hospital affiliated to Southeast University.

Support: There is no financial support.

Review Stage at time of this submission: Piloting of the study selection process.

Conflicts of interest:

None declared.

\section{Application of endometrial receptivity array (ERA) in embryo transfer cycles: a systematic review and meta-analysis}

Luo, R' Wang, J2; Shen, T3; Zhao, X4; Lu, J5.

Review question / Objective: To evaluate the impact of the personalized embryo transfer ( $\mathrm{pET}$ ) guided by endometrial receptivity analysis (ERA) on clinical outcomes in embryo transfer cycles.

Condition being studied: In vitro fertilization-embryo transfer cycles.

Information sources: We search Pubmed, EMBASE, and Web Of Science from inception to June 2021 for all studies comparing the clinical outcomes of the ERA group and the non-ERA group in patients undergoing embryo transfer cycles. Search terms for endometrial receptivity array or endometrial receptivity analysis (MeSH; live birth, pregnancy, miscarriage, implantation) and keywords 'pregnancy', 'live birth', 'miscarriage', or 'implantation' were combined with a search filter for studies related to humans. The abstracts of all studies identified were screened by two researchers (Luo $R$ and Wang JH). Any studies including data on ERA and clinical outcomes were read in full. Non-English language publications were translated if deemed relevant. Grey literature was also searched via the open grey website.

INPLASY registration number: This protocol was registered with the International Platform of Registered Systematic Review and Meta-Analysis Protocols (INPLASY) on 05 September 2021 and was last updated on 05 September 2021 (registration number INPLASY202190013).

\section{INTRODUCTION}

Review question / Objective: To evaluate the impact of the personalized embryo transfer ( $p E T)$ guided by endometrial receptivity analysis (ERA) on clinical outcomes in embryo transfer cycles.
Condition being studied: In vitro fertilization-embryo transfer cycles.

\section{METHODS}

Participant or population: Patients undergoing embryo transfer cycles. 
Intervention: ERA group: patients undergoing $P E T$ guided by ERA in embryo transfer cycles.

Comparator: Non-ERA group: patients undergoing standard embryo transfer cycles without ERA.

Study designs to be included: cohort study or randomized controlled trial study.

Eligibility criteria: Studies that compared the clinical outcomes of the ERA group and the non-ERA group in patients undergoing embryo transfer cycles. Studies published in abstract form were also included if the extraction of data was possible. Case reports, case series and review articles will be excluded.

Information sources: We search Pubmed, EMBASE, and Web Of Science from inception to June 2021 for all studies comparing the clinical outcomes of the ERA group and the non-ERA group in patients undergoing embryo transfer cycles. Search terms for endometrial receptivity array or endometrial receptivity analysis (MeSH; live birth, pregnancy, miscarriage, implantation) and keywords 'pregnancy', 'live birth', 'miscarriage', or 'implantation' were combined with a search filter for studies related to humans. The abstracts of all studies identified were screened by two researchers (Luo $R$ and Wang JH). Any studies including data on ERA and clinical outcomes were read in full. Non-English language publications were translated if deemed relevant. Grey literature was also searched via the open grey website.

Main outcome(s): Clinical pregnancy rate is the number of patients with one or more gestational sac containing at least one embryo with heartbeat per embryo transfer. The LBR is the number of deliveries that resulted in at least one live birth per embryo transfer. The miscarriage rate is the number of spontaneous pregnancy losses in which a gestational sac or sacs was previously observed, per number of clinical pregnancies. The implantation rate is the number of gestational sacs observed by vaginal ultrasound at the fifth gestational week divided by the number of embryos transferred. The ongoing pregnancy rate is the number of a positive pregnancy beyond 20 weeks gestation confirmed by ultrasound with fetal heart activity divided per embryo transfer.

Quality assessment / Risk of bias analysis: Quality of randomized controlled trials were assessed using the Cochrane risk assessment tool. Each study was qualified as High, Low, or Unclear risk of bias for each item. Quality of the cohort studies were assessed using the NewcastleOttawa Scale method. Scores $\geq 7$ is considered high quality and all other scores were considered low quality. Publication bias was not assessed if there are less than 10 studies in the meta-analysis.

Strategy of data synthesis: Two investigators will independently collect the data using a data extraction form and compare the final result with another person. If there is any disagreements between the two reviews, a third reviewer will solved the problem through discussion with.

Subgroup analysis: Patients with euploid embryo transfer cycles and donor cycles will be independently analyzed.

Sensitivity analysis: A sensitivity analysis was conducted using the leave-one-out approach if there was high heterogeneity between studies.

Language: No language ristrictions.

Country(ies) involved: China.

Keywords: Endometrial receptivity array, clinical outcomes, embryo transfer.

Contributions of each author:

Author 1 - Rong Luo.

Author 2 - Jiahui Wang.

Author 3 - Tao Shen.

Author 4 - Xia Zhao.

Author 5 - Jinchun Lu.

Author 6 - Yuanjiao Liang. 